\title{
FROM ELECTROSTATICS TO ALMOST OPTIMAL NODAL SETS FOR POLYNOMIAL INTERPOLATION IN A SIMPLEX*
}

\author{
J. S. HESTHAVEN ${ }^{\dagger}$
}

\begin{abstract}
The electrostatic interpretation of the Jacobi-Gauss quadrature points is exploited to obtain interpolation points suitable for approximation of smooth functions defined on a simplex. Moreover, several new estimates, based on extensive numerical studies, for approximation along the line using Jacobi-Gauss-Lobatto quadrature points as the nodal sets are presented.

The electrostatic analogy is extended to the two-dimensional case, with the emphasis being on nodal sets inside a triangle for which two very good matrices of nodal sets are presented. The matrices are evaluated by computing the Lebesgue constants and they share the property that the nodes along the edges of the simplex are the Gauss-Lobatto quadrature points of the Chebyshev and Legendre polynomials, respectively. This makes the resulting nodal sets particularly well suited for integration with conventional spectral methods and supplies a new nodal basis for $h-p$ finite element methods.
\end{abstract}

Key words. polynomial interpolation, Lebesgue constants, Jacobi polynomials, triangular elements, spectral methods

AMS subject classifications. 41A10, 65D05, 41A63, 78A30

PII. S003614299630587X

1. Introduction. In 1885 , Stieltjes $[1,2]$ revealed a remarkable connection between Jacobi polynomials and electrostatics by asking the following question.

Problem: Let two unit mass charges, $p>0$ and $q>0$, be held fixed at the positions $x= \pm 1$. Assume also that $N$ unit mass charges, positioned at $x_{1}, \ldots, x_{N}$, are allowed to move freely along the line connecting the two endpoint charges. What is the position of the charges that minimizes the electrostatic energy

$$
W\left(x_{1}, \ldots, x_{N}\right)=-\sum_{i=1}^{N}\left(p \log \left|x_{i}+1\right|+q \log \left|x_{i}-1\right|+\frac{1}{2} \sum_{\substack{j=1 \\ j \neq i}}^{N} \log \left|x_{i}-x_{j}\right|\right) .
$$

He continued by showing that the energy is minimized when $x_{1}, \ldots, x_{N}$ are given as the zeros of the Jacobi polynomial, $P_{N}^{\alpha, \beta}(x)$, with $\alpha=2 p-1$ and $\beta=2 q-1$. In 1939, Szegö [3] showed that this minimum is indeed the unique global minimum. Additionally, he showed that the Hermite and Laguerre polynomials also may be obtained through an electrostatic approach. Consequently, it was established that the Gauss quadrature points of the classical orthogonal polynomials can be determined as the steady state, minimum energy solution to a problem of electrostatics.

The symmetric Jacobi polynomials, $P_{N}^{\alpha, \alpha}(x)=P_{N}^{\alpha}(x)$, play a particularly important role in many areas of numerical analysis such as linear algebra and approximation theory. In particular, the special case of $P_{N}^{-1 / 2}(x)=T_{N}(x)$, known as the Chebyshev polynomials of the first kind, is used extensively for approximation of smooth func-

* Received by the editors July 1, 1996; accepted for publication (in revised form) December 23, 1996. This work was supported by NSF grant ASC-9504002 and by DOE grant DE-FG02-95ER25239.

http://www.siam.org/journals/sinum/35-2/30587.html

${ }^{\dagger}$ Division of Applied Mathematics, Brown University, Box F, Providence, RI 02912 (jansh@ cfm.brown.edu). 
tions. Indeed, the successful application of spectral methods for the solution of partial differential equations is based mainly on the superior approximation properties of the Chebyshev polynomials (see, e.g., [4]).

The apparent connection between steady state, minimum energy solutions to problems of electrostatics and polynomials, well suited for the approximation of smooth functions, is the main inspiration of the present work.

The question we attempt to address is whether it is possible to formulate an electrostatic problem in a simplex, the minimum energy, steady state solution of which leads to the specification of polynomials suitable for approximating smooth functions defined on the simplex.

We shall focus our attention on nodal sets and polynomial interpolation in an equilateral triangle. Such nodal sets are at the heart of the specification of highorder element methods, e.g., the $h-p$ finite element method [5] or spectral element methods [6]. Also, for the construction of diffeomorphic mapping functions, using transfinite blending functions, does the specification of high-order shape functions play an important role?

In the present work we will restrict our attention to nodal sets which share certain properties. In order for the nodal sets to be useful in connection with highorder element methods, it is important that the nodal sets include nodes along the boundaries of the simplex. In particular, along the line, this implies that the endpoints should be included in the nodal set. This allows for imposing boundary conditions and enforcing continuity between elements. In the two-dimensional case the picture is a little more complicated. Our aim is to obtain nodal sets that can be integrated with more conventional spectral methods or provide a fundamental building block for a high-order element method. In the latter case, the actual specification of the points along the edges of the simplex is less important since all elements are similar, such that enforcing continuity becomes a straightforward procedure. However, in relation to spectral methods the picture is a little more complicated. Traditional spectral methods in more than one dimension are based on tensor-products of one-dimensional approximations, with the most commonly used nodal sets being based on Gauss-Lobatto quadrature points of Chebyshev or Legendre polynomials. This has the additional advantage that Gauss quadrature rules exist for integration along the edges. Based on these observations we have chosen to restrict our attention to nodal sets in the simplex which share the property that the nodes along the edges are specified as Gauss-Lobatto quadrature points of symmetric Jacobi polynomials. Moreover, we require that the onedimensional edge polynomial is of the same order as the global multidimensional polynomial.

The remaining part of the paper is organized as follows. In section 2 we recall a few results from approximation theory important to the present work and we introduce the Lebesgue constant as a measure of the quality of the interpolating polynomial. Section 3 is devoted to interpolation of functions defined along the line. The known estimates of the Lebesgue constant for approximation using symmetric Jacobi polynomials are briefly reviewed and several new estimates, based on extensive numerical studies, using the symmetric Jacobi-Gauss-Lobatto quadrature points as nodal sets, are conjectured. In section 4 we turn to the problem of constructing highorder interpolating polynomials in the triangle through the solution of a problem of electrostatics. Section 5 contains a short discussion and concluding remarks. In the appendices we give the barycentric coordinates of two nodal matrices well suited for interpolation on a triangular domain. 
2. General concepts and notation. Throughout this paper we shall be concerned with the distribution of nodes in an $m$-dimensional simplex, $\mathrm{S}^{m} \subset \mathrm{R}^{m}$, leading to an almost optimal polynomial interpolation as measured through the Lebesgue constant. For the sake of simplicity, we restrict our analysis to the one-dimensional case, $m=1$, and the two-dimensional case, $m=2$, with the emphasis on $\mathrm{S}^{2}$ being an equilateral triangle.

We define the space of $n$-degree polynomials in $m$ variables, $\mathrm{P}_{n}^{m}$, such that the dimension of the approximation space is

$$
\operatorname{dim} \mathrm{P}_{n}^{m} \equiv N_{n}^{m}=\left(\begin{array}{c}
m+n \\
m
\end{array}\right),
$$

being the minimum space in which $\mathrm{P}_{n}^{m}$ may be complete [7]. Let us also introduce the nodal set, $\Pi_{n}^{m}=\left(\boldsymbol{x}_{1}, \ldots, \boldsymbol{x}_{N}\right)$, where the nodal points, or collocation points, are termed $\boldsymbol{x}_{i} \in \mathrm{S}^{m}$. Here, and in the following, we will use $N=N_{n}^{m}$ to simplify the notation unless clarification is deemed necessary.

Interpolation of smooth functions, $f\left[\mathrm{~S}^{m}\right]: \mathrm{R}^{m} \rightarrow \mathrm{R}$, where $f\left[\mathrm{~S}^{m}\right] \in C\left[\mathrm{~S}^{m}\right]$ and $f \in$ $\mathrm{H}$ is square integrable and belongs to the Hilbert space, $\mathrm{H}$, can now be viewed as, for a given $\Pi_{n}^{m}$, finding the polynomial $\mathcal{I}_{n}^{m} f \in \mathrm{P}_{n}^{m}$ such that $\forall \boldsymbol{x}_{i} \in \mathrm{S}^{m}, \mathcal{I}_{n}^{m} f\left(\boldsymbol{x}_{i}\right)=f\left(\boldsymbol{x}_{i}\right)$, where we have introduced the projector, $\mathcal{I}_{n}^{m}\left[\mathrm{~S}^{m}\right]: \mathrm{H} \rightarrow \mathrm{P}_{n}^{m}$. The solution to this problem is obtained by introducing the complete polynomial basis, $p_{i}(\boldsymbol{x}) \in \mathrm{P}_{n}^{m}$ and $\mathrm{P}_{n}^{m}=\operatorname{span}\left\{p_{i}(\boldsymbol{x})\right\}_{i=1}^{N}$, such that $\forall i \in[1, \ldots, N]: f\left(\boldsymbol{x}_{i}\right)=\sum_{j=1}^{N} a_{j} p_{j}\left(\boldsymbol{x}_{i}\right)$. Finding the expansion coefficients, $a_{j}$, is accomplished by solving the linear problem

$$
\left[\begin{array}{ccc}
p_{1}\left(\boldsymbol{x}_{1}\right) & \ldots & p_{N}\left(\boldsymbol{x}_{1}\right) \\
\vdots & & \vdots \\
p_{1}\left(\boldsymbol{x}_{N}\right) & \ldots & p_{N}\left(\boldsymbol{x}_{N}\right)
\end{array}\right]\left[\begin{array}{c}
a_{1} \\
\vdots \\
a_{N}
\end{array}\right]=\left[\begin{array}{c}
f\left(\boldsymbol{x}_{1}\right) \\
\vdots \\
f\left(\boldsymbol{x}_{N}\right)
\end{array}\right],
$$

or $\operatorname{VDM} \boldsymbol{a}=\boldsymbol{f}$, where the matrix is denoted $\operatorname{VDM}=\operatorname{VDM}\left(\boldsymbol{x}_{1}, \ldots, \boldsymbol{x}_{N}\right)$ in recognition of the fact that in the one-dimensional case it equals the Vandermonde matrix if $p_{j}\left(x_{i}\right)=\left(x_{i}\right)^{j-1}$. Existence and uniqueness of the interpolating polynomial is guaranteed if and only if the Vandermonde determinant, $|\mathrm{VDM}|$, is different from zero.

In the one-dimensional case this is ensured if the nodal points are distinct. However, for $m \geq 2$ no such simple results exist. A geometric characterization of distributions of points in $S^{m}$ ensuring a nonzero determinant is given in [8]. However, these considerations lead to sufficient conditions only and are certainly not necessary.

If we simply assume that $|\mathrm{VDM}| \neq 0$, we may express the polynomial approximation, $\mathcal{I}_{n}^{m} f(\boldsymbol{x}) \in \mathrm{P}_{n}^{m}$, using interpolating Lagrange polynomials, $L_{i}\left(\Pi_{n}^{m}, \boldsymbol{x}\right) \in \mathrm{P}_{n}^{m}$, with the property $L_{i}\left(\Pi_{n}^{m}, \boldsymbol{x}_{j}\right)=\delta_{i j}$. Thus, the polynomial interpolation is given as

$$
\mathcal{I}_{n}^{m} f(\boldsymbol{x})=\sum_{i=1}^{N} f\left(\boldsymbol{x}_{i}\right) L_{i}\left(\Pi_{n}^{m}, \boldsymbol{x}\right)
$$

This relation is naturally true for any smooth $f(\boldsymbol{x}) \in \mathrm{H}$, and in particular for the basis, $p_{i}(\boldsymbol{x})$, itself. Consequently, the interpolating Lagrange polynomials may in general be found as the solution to the dual interpolation problem

$$
\left[\begin{array}{ccc}
p_{1}\left(\boldsymbol{x}_{1}\right) & \ldots & p_{1}\left(\boldsymbol{x}_{N}\right) \\
\vdots & & \vdots \\
p_{N}\left(\boldsymbol{x}_{1}\right) & \ldots & p_{N}\left(\boldsymbol{x}_{N}\right)
\end{array}\right]\left[\begin{array}{c}
L_{1}\left(\Pi_{n}^{m}, \boldsymbol{x}\right) \\
\vdots \\
L_{N}\left(\Pi_{n}^{m}, \boldsymbol{x}\right)
\end{array}\right]=\left[\begin{array}{c}
p_{1}(\boldsymbol{x}) \\
\vdots \\
p_{N}(\boldsymbol{x})
\end{array}\right]
$$


through which, using Cramer's rule, we obtain the direct solution

$$
L_{i}\left(\Pi_{n}^{m}, \boldsymbol{x}\right)=\frac{\left|\operatorname{VDM}\left(\boldsymbol{x}_{1}, \ldots, \boldsymbol{x}_{i-1}, \boldsymbol{x}, \boldsymbol{x}_{i+1}, \ldots, \boldsymbol{x}_{N}\right)\right|}{\left|\operatorname{VDM}\left(\boldsymbol{x}_{1}, \ldots, \boldsymbol{x}_{N}\right)\right|} .
$$

The solution of Vandermonde systems, (1), is notoriously difficult due to exponentiallike growth of the condition number. Therefore, direct solution of the linear system, (1), should only be done for cases where no explicit formula for the Lagrange polynomials is known, however complicated it may be. If, indeed, it is necessary to solve the Vandermonde problem, great care must be exercised for large values of $N$. We refer to [9] for a discussion of this topic and for references to dealing with the accurate solution of Vandermonde systems.

We equip the polynomial space, $\mathrm{P}_{n}^{m}$, with the supremum-norm, $\|\cdot\|$, and introduce the measures

$$
\left\|\mathcal{I}_{n}^{m}\right\|=\sup _{f \neq 0} \frac{\left\|\mathcal{I}_{n}^{m} f\right\|_{\infty}}{\|f\|_{\infty}},\|f\|_{\infty}=\max _{\mathbf{x} \in \mathrm{S}^{m}}|f(\boldsymbol{x})|
$$

The interpolation error is uniformly bounded from below by the best approximation as

$$
\left\|f(\boldsymbol{x})-p^{*}(\boldsymbol{x})\right\|_{\infty} \leq\left\|f(\boldsymbol{x})-\mathcal{I}_{n}^{m} f(\boldsymbol{x})\right\|_{\infty},
$$

where $p^{*}(\boldsymbol{x})$ signifies the best approximating polynomial ensured to exist since $f$ is assumed to be continuous [10]. For $m=1$, the rate of convergence of $p^{*}(x)$ is guaranteed to be at least geometric in $N$ through Jackson's theorem. For $m \geq 2$, no such results exist for the simplex.

Unfortunately, the determination of $p^{*}(\boldsymbol{x})$ is, even for $m=1$, an unsolved problem, in the general case and for $m \geq 2$, virtually nothing is known. A powerful way of estimating the quality of alternative approximations as compared to $p^{*}(\boldsymbol{x})$ appears as a result of the following theorem.

THEOREM 2.1 (Lebesgue). Assume that $f\left[\mathrm{~S}^{m}\right] \in C\left[\mathrm{~S}^{m}\right]$ and that we consider the nodal set, $\Pi_{n}^{m}$; then

$$
\left\|f(\boldsymbol{x})-\mathcal{I}_{n}^{m} f(\boldsymbol{x})\right\|_{\infty} \leq\left[1+\Lambda\left(\Pi_{n}^{m}\right)\right]\left\|f(\boldsymbol{x})-p^{*}(\boldsymbol{x})\right\|_{\infty},
$$

where

$$
\Lambda\left(\Pi_{n}^{m}\right)=\left\|\mathcal{I}_{n}^{m}\right\|=\max _{\mathbf{x} \in \mathrm{S}^{m}} \sum_{i=1}^{N}\left|L_{i}\left(\Pi_{n}^{m}, \boldsymbol{x}\right)\right|
$$

is termed the Lebesgue constant.

Consequently, by computing the Lebesgue constant we obtain a measure of how close the approximation is to the best polynomial approximation. It is noteworthy that the value of the Lebesgue constant depends solely on the interpolating Lagrange polynomials and, therefore, is determined completely by the nodal set, $\Pi_{n}^{m}$.

For interpolation of smooth functions on $\mathrm{S}^{1}$, we learn from Theorem 2.1 that convergence can be expected only when the function is sufficiently smooth such that the approximation error decays faster than the growth of the Lebesgue constant. Another motivation for searching for nodal sets resulting in small Lebesgue constants is that due to finite precision of digital computers, given through the machine accuracy, $\varepsilon_{M}$, one can only expect valid results when $\left[\Lambda\left(\Pi_{n}^{m}\right)\right]^{-1} \gg \varepsilon_{M}$, independent 
of the smoothness of the function being approximated. It is therefore of significant importance to specify methods for obtaining nodal sets resulting in small and slowly growing Lebesgue constants. The study of this is the subject of the remaining part of the paper.

3. Interpolation points in $\mathbf{S}^{\mathbf{1}}$ using electrostatics. The interpolation problem in $S^{1}$ has received extensive attention in the past, and we shall not attempt to fully account for the large number of results, but rather selectively present the relevant theory. For a general introduction we refer to $[10,11,12]$.

For $m=1$, we introduce the polynomial space, $\mathrm{P}_{n}^{1}=\operatorname{span}_{\tilde{\Pi}_{n}}\left\{p_{i}(x) \mid p_{i}(x)=x^{i-1}\right\}_{i=1}^{n+1}$, since $N_{n}^{1}=N=n+1$, and the corresponding nodal set, $\tilde{\Pi}_{n}^{1}=\left\{x_{i}\right\}_{i=1}^{n+1}$, where it is assumed that the nodes are ordered as $a=x_{1}<\cdots<x_{n+1}=b$.

For $\mathrm{S}^{1}=[a, b]$, VDM becomes the regular Vandermonde matrix with the determinant given as

$$
\left|\operatorname{VDM}\left(x_{1}, \ldots x_{n+1}\right)\right|=\prod_{i=1}^{n+1} \prod_{j=i+1}^{n+1}\left(x_{i}-x_{j}\right),
$$

and, consequently, the interpolating Lagrange polynomials exist and are unique provided only that the nodes are distinct. Moreover, the interpolating Lagrange polynomial in $S^{1}$ is given in explicit form as

$$
L_{i}\left(\tilde{\Pi}_{n}^{1}, x\right)=\prod_{\substack{j=1 \\ j \neq i}}^{n+1} \frac{x-x_{j}}{x_{i}-x_{j}} .
$$

If no further constraints are imposed on the position of the nodes, the nodal configuration leading to an optimal Lebesgue constant is nonunique [13].

However, if we restrict the permissible nodal sets by requiring the endpoints to be fixed at $x_{1}=-1$ and $x_{n+1}=1$, the setting becomes considerably more firm. Sets endowed with this constraint are known as canonical sets. Any degree $N_{n}^{1}$ nodal set, $\tilde{\Pi}_{n}^{1}=\left[a=x_{1}<\cdots<x_{n+1}=b\right]$, may be mapped to the canonical nodal set, $\Pi_{n}^{1}$, through a linear mapping. Moreover, since the Lagrange polynomials are invariant under the linear mapping, i.e., $\Lambda\left(\Pi_{n}^{1}\right) \leq \Lambda\left(\tilde{\Pi}_{n}^{1}\right)$, it is sufficient to consider the properties of the canonical nodal sets to which we shall mainly direct our attention below.

The equioscillatory property of the Lebesgue functions, defined as

$$
\lambda\left(\Pi_{n}^{m}, \boldsymbol{x}\right)=\sum_{i=1}^{N}\left|L_{i}\left(\Pi_{n}^{m}, \boldsymbol{x}\right)\right|,
$$

characterizing the optimal canonical nodal set, was first conjectured in the famous Bernstein-Erdös conjecture and later proved in $[14,15]$. This result establishes existence and uniqueness of the optimal set and, among other properties, shows that the optimal nodal set, $\Pi_{n}^{1}$, is symmetric around $x=0$. A numerical procedure for computing the optimal nodal set in $\mathrm{S}^{1}$ is given in [16].

Bounds for the Lebesgue constant of the optimal canonical nodal set were first obtained in [17].

THEOREM 3.1. For all permissible nodal sets, $\Pi_{n}^{1}$, and all $n$ there exists a positive constant, $c$, such that

$$
\Lambda\left(\Pi_{n}^{1}\right)>\frac{2}{\pi} \log (n+1)-c .
$$


This was later refined considerably [12] as the following theorem.

THEOREM 3.2. The Lebesgue constant, $\Lambda\left(\Pi_{n}^{1}\right)$, for the optimal canonic nodal set, $\Pi_{n}^{1}$, in $\mathrm{S}^{1}$ is bounded as

$c\left(\frac{\log \log (n+1)}{\log (n+1)}\right)^{2}>\Lambda\left(\Pi_{n}^{1}\right)-\frac{2}{\pi} \log (n+1)-\chi> \begin{cases}\frac{\pi}{18(n+1)^{2}}+\mathcal{O}\left(\frac{1}{(n+1)^{4}}\right) & \text { for } n \text { odd, } \\ -\frac{2}{\pi(n+1)}+\mathcal{O}\left(\frac{1}{(n+1)^{2}}\right) & \text { for } n \text { even, }\end{cases}$

where

$$
\chi=\frac{2}{\pi}\left(\gamma+\log \frac{4}{\pi}\right)=0.52125162 \ldots
$$

and $\gamma=0.57721566 \ldots$ represents Euler's constant.

The value of the Lebesgue constant for various choices of nodal sets has been given much attention in the past. However, as we have discussed previously, we are primarily interested in nodal sets obtained as Gauss-Lobatto quadrature points for the symmetric Jacobi polynomials due to their extensive use in connection with spectral methods. We have therefore chosen to restrict the discussion below to results obtained for the Jacobi polynomials only.

Equally distributed nodal sets. For reasons of comparison, let us, however, first focus our attention on the interpolation problem at an equidistant grid and introduce the equally distributed nodal set, $\Pi_{n}^{\mathrm{Eq}}=\left\{x_{i} \mid x_{i}=-1+2(i-1) / n\right\}_{i=1}^{n+1}$, with the associated Lebesgue constant, $\Lambda_{n}^{\mathrm{Eq}}=\Lambda\left(\Pi_{n}^{\mathrm{Eq}}\right)$. The well-known Runge phenomenon associated with interpolation at an equidistant grid manifests itself through a very rapid growth of the Lebesgue constant [18, 19, 20]

THEOREM 3.3. The Lebesgue constant, $\Lambda_{n}^{\mathrm{Eq}}$, for interpolation in $\mathrm{S}^{1}$ with an equally distributed nodal set, $\Pi_{n}^{\mathrm{Eq}}$, is bounded for $n \geq 1$ as

$$
\frac{2^{n-2}}{n^{2}}<\Lambda_{n}^{\mathrm{Eq}}<\frac{2^{n+3}}{n}
$$

with the asymptotic estimate

$$
\Lambda_{n}^{\mathrm{Eq}} \simeq \frac{2^{n+1}}{e n(\log n+\gamma)} \quad \text { for } n \rightarrow \infty,
$$

where $\gamma$ represents Euler's constant.

Clearly, the very fast growth of $\Lambda_{n}^{\mathrm{Eq}}$ with $n$ confirms that interpolation in $\mathrm{S}^{1}$ using $\Pi_{n}^{\mathrm{Eq}}$ results in a very poor approximation for increasing $n$. Indeed, for $n \geq 65$, we find that $\mathcal{O}\left(\left(\Lambda_{n}^{\mathrm{Eq}}\right)^{-1}\right) \sim 10^{-16}$, rendering the approximation useless on most contemporary computers. For reasons of comparison, we list $\Lambda_{n}^{\mathrm{Eq}}$ for $n \in[1,24]$ in Table 1.

Chebyshev distributed nodal sets. Let us now turn to nodal sets associated with zeros of Jacobi polynomials and begin by considering the Cauchy remainder for polynomial interpolation

$$
f(x)-\mathcal{I}_{n}^{1} f(x)=\prod_{i=1}^{n+1}\left(x-x_{i}\right) \frac{1}{(n+1) !} \frac{d^{n+1} f(x)}{d x^{n+1}}
$$

indicating that a good choice for interpolation may be the nodal set minimizing $\left\|\prod_{i=1}^{n+1}\left(x-x_{i}\right)\right\|_{\infty}$. This uniquely determined nodal set, $\Pi_{n}^{\mathrm{CG}}$, is recognized as the 
TABLE 1

Lebesgue constants for various choices of nodal sets. Listed is the minimum Lebesgue constant, $\Lambda_{n}^{\mathrm{JGL}, \alpha}$, for the optimal value of $\alpha$ using the Jacobi-Gauss-Lobatto nodal set, $\Pi_{n}^{\mathrm{JGL}, \alpha}$. Also given are $\Lambda_{n}^{\mathrm{LGL}}$ and $\Lambda_{n}^{\mathrm{CGL}}$ representing the Lebesgue constants for the Legendre-Gauss-Lobatto, $\Pi_{n}^{\mathrm{LGL}}$, and the Chebyshev-Gauss-Lobatto, $\Pi_{n}^{\mathrm{CGL}}$, nodal sets. For comparison we also list the Lebesgue constants, $\Lambda_{n}^{\mathrm{Opt}}$, for the optimal nodal set [27] and the Lebesgue constant, $\Lambda_{n}^{\mathrm{Eq}}$, corresponding to an equally distributed nodal set, $\Pi_{n}^{\mathrm{Eq}}$.

\begin{tabular}{||r||c|c||c|c||c|c||}
\hline$n$ & $\alpha$ & $\Lambda_{n}^{\mathrm{JGL}, \alpha}$ & $\Lambda_{n}^{\mathrm{LGL}}$ & $\Lambda_{n}^{\mathrm{CGL}}$ & $\Lambda_{n}^{\mathrm{Opt}}$ & $\Lambda_{n}^{\mathrm{Eq}}$ \\
\hline 1 & All & 1.0000 & 1.0000 & 1.0000 & 1.0000 & 1.0000 \\
2 & All & 1.2500 & 1.2500 & 1.2500 & 1.2500 & 1.2500 \\
3 & 0.364636 & 1.4229 & 1.5000 & 1.6667 & 1.4229 & 1.6311 \\
4 & 0.390667 & 1.5595 & 1.6359 & 1.7988 & 1.5595 & 2.2078 \\
5 & 0.410978 & 1.6746 & 1.7786 & 1.9889 & 1.6722 & 3.1063 \\
6 & 0.420531 & 1.7709 & 1.8737 & 2.0826 & 1.7681 & 4.5493 \\
\hline 7 & 0.428904 & 1.8560 & 1.9724 & 2.2022 & 1.8516 & 6.9297 \\
8 & 0.433838 & 1.9301 & 2.0456 & 2.2747 & 1.9255 & 10.945 \\
9 & 0.438665 & 1.9973 & 2.1210 & 2.3619 & 1.9917 & 17.848 \\
10 & 0.441695 & 2.0575 & 2.1805 & 2.4210 & 2.0517 & 29.899 \\
11 & 0.444993 & 2.1129 & 2.2415 & 2.4894 & 2.1066 & 51.214 \\
12 & 0.446733 & 2.1636 & 2.2917 & 2.5393 & 2.1571 & 89.324 \\
\hline 13 & 0.448718 & 2.2109 & 2.3428 & 2.5957 & 2.2040 & 158.09 \\
14 & 0.450689 & 2.2545 & 2.3862 & 2.6388 & 2.2476 & 283.19 \\
15 & 0.451792 & 2.2958 & 2.4303 & 2.6867 & 2.2884 & 512.35 \\
16 & 0.453426 & 2.3341 & 2.4684 & 2.7247 & 2.3268 & 934.50 \\
17 & 0.453893 & 2.3707 & 2.5072 & 2.7664 & 2.3630 & 1716.4 \\
18 & 0.454938 & 2.4049 & 2.5412 & 2.8003 & 2.3973 & 3171.1 \\
\hline 19 & 0.456111 & 2.4375 & 2.5758 & 2.8371 & 2.4298 & 5889.4 \\
20 & 0.456752 & 2.4685 & 2.6065 & 2.8677 & 2.4608 & 10986. \\
21 & 0.457683 & 2.4980 & 2.6377 & 2.9008 & - & 20574. \\
22 & 0.459342 & 2.5260 & 2.6658 & 2.9288 & - & 38667. \\
23 & 0.459593 & 2.5532 & 2.6942 & 2.9587 & - & 72908. \\
24 & 0.459831 & 2.5792 & 2.7200 & 2.9844 & - & 137852. \\
\hline
\end{tabular}

Chebyshev-Gauss quadrature points, i.e., the zeros of the Chebyshev polynomial, $P_{n+1}^{-1 / 2}(x)=T_{n+1}(\cos \theta)=\cos ((n+1) \theta)$.

As realized by Stieltjes $[1,2]$, these points may be found as a solution to the electrostatic problem with $p=q=1 / 4$. Note, however, that this nodal set is not canonical since $\Pi_{n}^{\mathrm{CG}}=\left\{x_{i} \mid x_{i}=-\cos ((2 i-1) \pi / 2(n+1))\right\}_{i=1}^{n+1}$. The Lebesgue constant, $\Lambda_{n}^{\mathrm{CG}}=\Lambda\left(\Pi_{n}^{\mathrm{CG}}\right)$, for the Chebyshev-Gauss nodal set is given as [21, 22]

THEOREM 3.4. The Lebesgue constant, $\Lambda_{n}^{\mathrm{CG}}$, for interpolation using the ChebyshevGauss nodal set, $\Pi_{n}^{\mathrm{CG}}$, is bounded for $n \geq 1$ as

$$
\Lambda_{n}^{\mathrm{CG}}=\frac{2}{\pi} \log (n+1)+A+\alpha_{n},
$$

where

$$
A=\frac{2}{\pi}\left(\gamma+\log \frac{8}{\pi}\right) \quad \text { and } \quad 0<\alpha_{n}<\frac{\pi}{72(n+1)^{2}}
$$

The asymptotic behavior of $\Lambda_{n}^{\mathrm{CG}}$ is given by

$$
\Lambda_{n}^{\mathrm{CG}} \simeq \frac{2}{\pi} \log (n+1)+A+\frac{8}{\pi} \sum_{s=1}^{\infty} \frac{(-1)^{s+1} A_{s}}{[2(n+1)]^{2 s}}
$$


where

$$
A_{s}=\left(2^{2 s-1}-1\right)^{2} \frac{\pi^{2 s}}{2 s} \frac{B_{2 s}^{2}}{(2 s) !},
$$

and $B_{p}$ signifies the Bernoulli numbers.

Comparing with the optimal values of the Lebesgue constant given in Theorem 3.2 , is it clear that interpolation using $\Pi_{n}^{\mathrm{CG}}$ results in an approximation error being close to that of the optimal canonical nodal set.

However, if we consider the canonical Chebyshev-Gauss or extended ChebyshevGauss nodal set, $\Pi_{n}^{\mathrm{ECG}}$, obtained through the linear mapping

$$
\Pi_{n}^{\mathrm{ECG}}=\left\{x_{i} \mid x_{i}=-\frac{\cos \left(\frac{2 i-1}{2(n+1)} \pi\right)}{\cos \left(\frac{\pi}{2(n+1)}\right)}\right\}_{i=1}^{n+1},
$$

the Lebesgue constant, $\Lambda_{n}^{\mathrm{ECG}}=\Lambda\left(\Pi_{n}^{\mathrm{ECG}}\right)$, is bounded by [21].

THEOREM 3.5. The Lebesgue constant, $\Lambda_{n}^{\mathrm{ECG}}$, for interpolation using the extended Chebyshev-Gauss nodal set, $\Pi_{n}^{\mathrm{ECG}}$, is bounded for $n \geq 4$ as

$$
\Lambda_{n}^{\mathrm{ECG}}=\frac{2}{\pi} \log (n+1)+A-\frac{4}{3 \pi}+\beta_{n},
$$

where

$$
0<\beta_{n}<0.01\left[\log \left(\frac{n+1}{4}\right)\right]^{-1}
$$

By expressing the two constants in Theorems 3.4 and 3.5 as

$$
A-\chi=\frac{2}{\pi} \log 2 \quad \text { and } \quad A-\frac{4}{3 \pi}-\chi=\frac{2}{\pi}\left(\log 2-\frac{2}{3}\right)=0.01685801 \ldots,
$$

it becomes clear that both nodal sets are close to the optimal set for large values of $n$. However, in particular, the extended Chebyshev-Gauss nodal set, $\Pi_{n}^{\mathrm{ECG}}$, results in an approximation that is very close to that obtained using the optimal nodal set. Indeed, to the best of our knowledge, the extended Chebyshev-Gauss nodal set is the best-known nodal set with the position of the nodes given on closed form.

However, $\Pi_{n}^{\mathrm{ECG}}$ does not represent a nodal set associated with the zeros of a Jacobi polynomial. As discussed previously, this issue is important in connection with the solution of partial differential equations using spectral methods where highorder integration is required. Consequently, in such cases it is desirable that the nodal set be related to a Gauss quadrature formula, explaining why the extended Chebyshev-Gauss nodal set plays a less important role in this context.

Also, the Chebyshev-Gauss nodal set is less attractive for use in connection with spectral methods as the endpoints of the interval are not included, thereby making it hard to enforce boundary conditions. For these reasons, the most used nodal set for constructing approximate solutions to partial differential equations using spectral methods is the canonical Chebyshev-Gauss-Lobatto set obtained as the zeros of the polynomial, $\left(1-x^{2}\right) T_{n}^{\prime}(x)$, and given in closed form as $\Pi_{n}^{C G L}=\left(-1,\left\{x_{i}\right\}_{i=2}^{n}, 1\right)$ where $x_{i}=-\cos ((i-1) \pi / n)$. 
To return to the electrostatic analogy, we recall that the Jacobi-Gauss quadrature points appear from the original work. However, since the Jacobi-Gauss-Lobatto quadrature points are obtained as the interior zeros of the polynomial $\left(P_{n}^{\alpha}(x)\right)^{\prime}$ and using the identity for Jacobi polynomials [23],

$$
2 \frac{d}{d x} P_{n}^{\alpha}(x)=(n+1+2 \alpha) P_{n-1}^{\alpha+1}(x),
$$

we realize also that the Jacobi-Gauss-Lobatto quadrature points appear as solutions to the original electrostatic problem by using the relation $\alpha=2(p-1)$ and including the endpoints in the nodal set; e.g., using $p=q=\frac{3}{4}$ results in the Chebyshev-GaussLobatto nodal set $\left(\alpha=-\frac{1}{2}\right)$.

Estimation of the Lebesgue constant, $\Lambda_{n}^{\mathrm{CGL}}=\Lambda\left(\Pi_{n}^{\mathrm{CGL}}\right)$, for the ChebyshevGauss-Lobatto nodal set is done in [24], yielding the following result.

THEOREM 3.6. The Lebesgue constant, $\Lambda_{n}^{\mathrm{CGL}}$, using the Chebyshev-Gauss-Lobatto nodal set, $\Pi_{n}^{\mathrm{CGL}}$, is bounded as

$$
\begin{array}{ll}
\Lambda_{n}^{\mathrm{CGL}}<\Lambda_{n-1}^{\mathrm{CG}} & n \text { even } \\
\Lambda_{n}^{\mathrm{CGL}}=\Lambda_{n-1}^{\mathrm{CG}} & n \text { odd }
\end{array}
$$

where $\Lambda_{n}^{\mathrm{CG}}$ represents the Lebesgue constant obtained for the Chebyshev-Gauss nodal set, $\Pi_{n}^{\mathrm{CG}}$, as given in Theorem 3.4.

We immediately observe that in addition to the desirable property that the GaussLobatto nodal sets include the endpoints of $\mathrm{S}^{1}$, the Lebesgue constants are also uniformly bounded by those obtained using the Gauss nodal set.

The tight bound on $\Lambda_{n}^{\mathrm{CGL}}$ for $n$ being odd is possibly due to the observation [24] that $\Lambda_{n}^{\mathrm{CGL}}=\lambda\left(\Pi_{n}^{\mathrm{CGL}}, 0\right)$, where $\lambda\left(\Pi_{n}^{\mathrm{CGL}}, x\right)$ represents the Lebesgue function. Based on extensive numerical evidence, we conjecture as follows.

COnJeCture 3.1. The Lebesgue constant, $\Lambda_{n}^{\mathrm{CGL}}$, using the Chebyshev-GaussLobatto nodal set, $\Pi_{n}^{\mathrm{CGL}}$, is given as

$$
n \text { even }: \Lambda_{n}^{\mathrm{CGL}}=\lambda\left(\Pi_{n}^{\mathrm{CGL}}, \frac{\pi}{2 n}\right), n \rightarrow \infty .
$$

For $n$ being even, $x=0$ is always part of the nodal set as $x_{n / 2}$. Consequently, it is conjectured that the Lebesgue function attains its maximum value exactly between the center node, $x_{n / 2}$, and $x_{(n+2) / 2}$, since

$$
\frac{x_{(n+2) / 2}}{2}=-\frac{1}{2} \cos \left(\frac{\pi}{2} \frac{n+2}{n}\right)=\frac{1}{2} \sin \left(\frac{\pi}{n}\right) \sim \frac{\pi}{2 n}
$$

for large values of $n$. This result conforms well with the result that for $n$ being odd, the maximum of the Lebesgue function is also found exactly between the two center nodes, i.e., at $x=0$.

Values of the Lebesgue constant, $\Lambda_{n}^{\mathrm{CGL}}$, are given in Table 1 for $n \in[1,24]$, and in Fig. 1 we plot $\Lambda_{n}^{\text {CGL }}$ for large $n$, confirming the logarithmic dependence on $n$.

Legendre distributed nodal sets. Based on the general expression for the interpolating Lagrange polynomials, (2), it appears that the nodal set maximizing the Vandermonde determinant, |VDM|, could be expected to yield an interpolating polynomial with a small value of the Lebesgue constant. As shown in [25], this nodal set is given as $\Pi_{n}^{\mathrm{LGL}}=\left(-1,\left\{x_{i}\right\}_{i=2}^{n}, 1\right)$ where $\left\{x_{i}\right\}_{i=2}^{n}=\left\{x_{i} \mid\left(P_{n}^{0}\left(x_{i}\right)\right)^{\prime}=0\right\}_{i=2}^{n}$, i.e., the extrema of $P_{n}^{0}(x)$ also known as the Legendre polynomial. This nodal set, $\Pi_{n}^{\mathrm{LGL}}$, is known as the Legendre-Gauss-Lobatto or the Fekete/Fejér nodal set. 


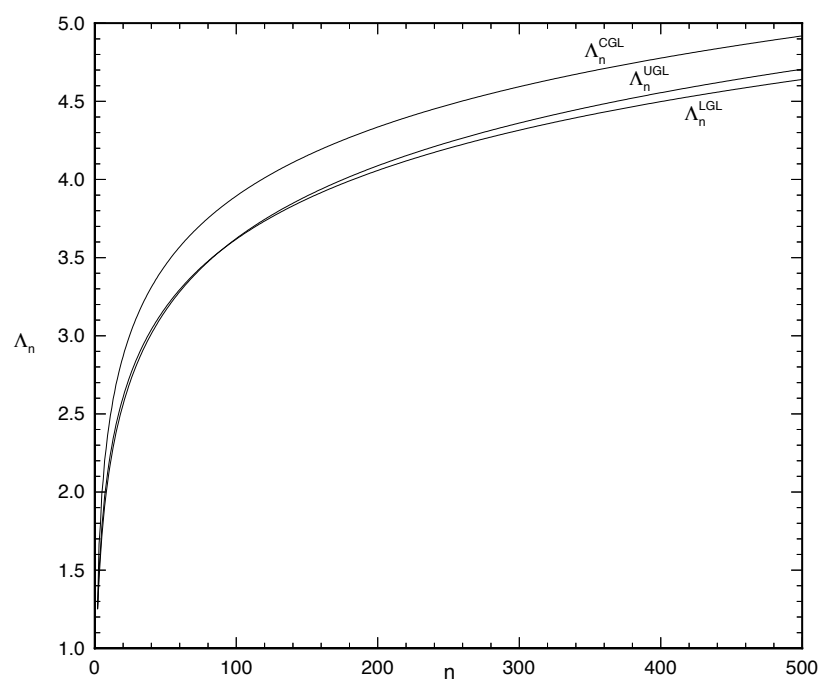

FIG. 1. The Lebesgue constants as a function of $n$ for various choices of Jacobi-Gauss-Lobatto nodal sets. Shown are the Lebesgue constants for the Legendre polynomials, $\Lambda_{n}^{\mathrm{LGL}}$, and for Chebyshev polynomials of the first, $\Lambda_{n}^{\mathrm{CGL}}$, and second kind, $\Lambda_{n}^{\mathrm{UGL}}$, respectively.

Estimates for the Lebesgue constant, $\Lambda_{n}^{\mathrm{LGL}}=\Lambda\left(\Pi_{n}^{\mathrm{LGL}}\right)$, are sparse, the problem being that no explicit formula for the nodal set is known. The first estimate is possibly given in [25] as $\Lambda_{n}^{\mathrm{LGL}} \leq \sqrt{n+1}$, which is also obtained simply by applying the Cauchy-Schwartz inequality and the fact $\sum_{i=1}^{n+1}\left|L_{i}\left(\Pi_{n}^{\mathrm{LGL}}, x\right)\right|^{2} \leq 1$. This, however, is very pessimistic, as evidenced by the conjecture in [13] that $\Lambda_{n}^{\mathrm{LGL}}<\Lambda_{n}^{\mathrm{CG}}$ and the estimate $\Lambda_{n}^{\mathrm{LGL}} \leq c \log (n+1)$ put forward in [26], although the constant, $c$, remains undetermined. Based on numerical experiments, we conjecture the following.

CONJECTURE 3.2. The Lebesgue constant, $\Lambda_{n}^{\mathrm{LGL}}$, using the Legendre-GaussLobatto nodal set, $\Pi_{n}^{\mathrm{LGL}}$, is bounded as

$$
\Lambda_{n}^{\mathrm{LGL}} \leq \frac{2}{\pi} \log (n+1)+0.685
$$

and

$$
\begin{array}{ll}
\Lambda_{n}^{\mathrm{LGL}}=\lambda\left(\Pi_{n}^{\mathrm{LGL}}, 0\right) & n \text { odd }, \\
\Lambda_{n}^{\mathrm{LGL}} \simeq \lambda\left(\Pi_{n}^{\mathrm{LGL}}, \frac{\pi}{2 n}\right) & n \text { even. }
\end{array}
$$

This conjecture is consistent with those put forward in [13] and does indeed confirm that $\Lambda_{n}^{\mathrm{LGL}}<\Lambda_{n}^{\mathrm{CG}}$ uniformly in $n$. Additionally, we also find $\Lambda_{n}^{\mathrm{LGL}}<\Lambda_{n}^{\mathrm{CGL}}$ uniformly in $n$, as is illustrated in Fig. 1. Numerical values of $\Lambda_{n}^{\mathrm{LGL}}$ are given in Table 1 for $n \in[1,24]$, supporting the conjecture.

Jacobi distributed nodal sets. Let us finally focus our attention on the general case, about which very little is known. If we first consider the Jacobi-Gauss nodal set, $\Pi_{n}^{\mathrm{JG}, \alpha}$, being given as $\Pi_{n}^{\mathrm{JG}, \alpha}=\left\{x_{i} \mid P_{n+1}^{\alpha}\left(x_{i}\right)=0\right\}_{i=1}^{n+1}$, the most general result is [3]. 
THEOREM 3.7. The Lebesgue constant, $\Lambda_{n}^{\mathrm{JG}, \alpha}$, using the Jacobi-Gauss nodal set, $\Pi_{n}^{\mathrm{LGL}}$, scales as

$$
\begin{aligned}
\Lambda_{n}^{\mathrm{JG}, \alpha} & \sim \mathcal{O}(\log (n+1)) & & \alpha \leq-\frac{1}{2}, \\
\Lambda_{n}^{\mathrm{JG}, \alpha} & \sim \mathcal{O}(\sqrt{n+1}) & & \alpha>-\frac{1}{2} .
\end{aligned}
$$

Unfortunately, no such general results exist when considering the nodal sets, $\Pi_{n}^{\mathrm{JGL}, \alpha}$, based on the Jacobi-Gauss-Lobatto quadrature points, given as $\Pi_{n}^{\mathrm{JGL}, \alpha}=$ $\left(-1,\left\{x_{i}\right\}_{i=2}^{n}, 1\right)$ where we have $\left\{x_{i}\right\}_{i=2}^{n}=\left\{x_{i} \mid\left(P_{n}^{\alpha}\left(x_{i}\right)\right)^{\prime}=0\right\}_{i=2}^{n}$. We have previously discussed the two special cases of the Chebyshev-Gauss-Lobatto nodal set, $\alpha=-1 / 2$, and the Legendre-Gauss-Lobatto nodal set, $\alpha=0$. An interesting question arises: among all $\Pi_{n}^{\mathrm{JGL}, \alpha}$, which value of $\alpha$ minimizes the Lebesgue constant, $\Lambda_{n}^{\mathrm{JGL}, \alpha}$, for a given $n$ ?

In attempting to address this question, we give in Table 1 the value of $\alpha$ leading to the minimum Lebesgue constant, $\Lambda_{n}^{\mathrm{JGL}, \alpha}$, among all the nodal sets based on the Jacobi-Gauss-Lobatto quadrature points, $\Pi_{n}^{\mathrm{JGL}, \alpha}$, for various values of $n$. By comparing this with the Lebesgue constants, $\Lambda_{n}^{\text {Opt }}$, for the optimal nodal set [27], is it clear that the computed Jacobi-Gauss-Lobatto nodal sets are very close to the optimal nodal set? We observe that the value of $\alpha$ leading to the optimal nodal set varies with $n$, although it seems that it asymptotes towards a fixed value around $\alpha \simeq 0.5$. This tendency is found to be maintained for $n \leq 60$. However, we have been unable to continue the computation beyond this point, as the computation of the optimal value of $\alpha$ becomes prohibitively expensive due to the many required computations of the corresponding Lebesgue constant.

The value of $\alpha=1 / 2$ corresponds to a nodal set, $\Pi_{n}^{\mathrm{UGL}}$, based on the GaussLobatto quadrature points for the Chebyshev polynomial of the second kind being given as $U_{n}(\cos (\theta))=\sin ((n+1) \theta) / \sin \theta$. Unfortunately, as we will see shortly, the hypothesis that $\Pi_{n}^{\mathrm{UGL}}$ is close to the optimal nodal set is only valid for small $n$. We recall that the nodal set is given in explicit form as $\Pi_{n}^{\mathrm{UGL}}=\left\{-1,\left\{x_{i}\right\}_{i=2}^{n}, 1\right\}$ where $x_{i}=-\cos ((2 i-1) \pi / 2(n+1))$.

We are not aware of any results estimating the Lebesgue constant, $\Lambda_{N}^{\mathrm{UGL}}=$ $\Lambda\left(\Pi_{n}^{\mathrm{UGL}}\right)$, using the Gauss-Lobatto nodal set of the Chebyshev polynomials of the second kind. Based on extensive numerical studies, we conjecture the following.

CONJECTURE 3.3. The Lebesgue constant, $\Lambda_{n}^{\mathrm{UGL}}$, using the Chebyshev-GaussLobatto nodal set, $\Pi_{n}^{\mathrm{UGL}}$, is bounded as

$$
\Lambda_{n}^{\mathrm{UGL}} \leq \frac{2}{\pi} \log (n+1)+\beta_{n},
$$

with

$$
0<\beta_{n}<0.525 f(n+1),
$$

where $f$ is a weak function of $n$ behaving asymptotically as

$$
n \rightarrow \infty: \frac{f(n)}{\log n} \rightarrow \infty \quad, \quad \frac{f(n)}{\sqrt{n}} \rightarrow 0 .
$$

We have been unable to estimate the unknown function, $f(n)$, more accurately. However, numerical studies indicate that, asymptotically, $f(n) \simeq n^{\alpha}$, where $\alpha \sim$ $\mathcal{O}(0.1)$. 


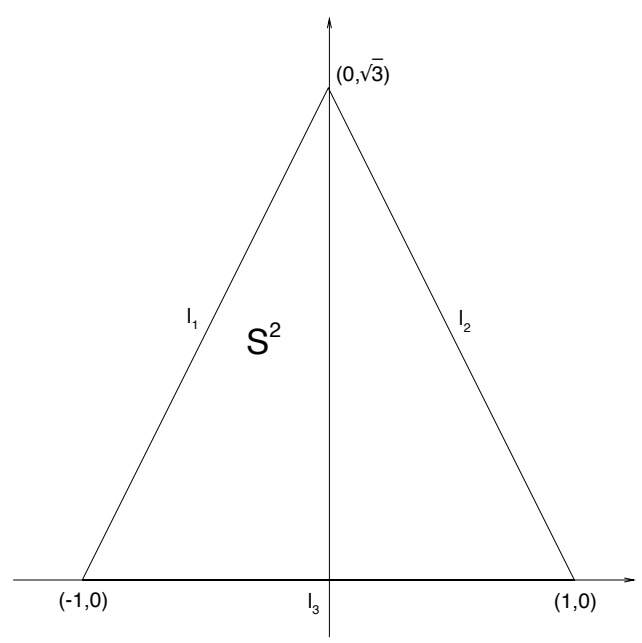

FiG. 2. The standard equilateral triangle, $\mathrm{S}^{2}$.

We observe that for small values of $n$, the nodal sets associated with Chebyshev polynomials of the second kind results in a Lebesgue constant that is very close to the optimal one as given in Theorem 3.2. However, the slow growth of $\Lambda_{n}^{\mathrm{UGL}}$ with $n$ renders this property invalid for increasing $n$. In Fig. 1 we compare $\Lambda_{n}^{\mathrm{UGL}}$ with $\Lambda_{n}^{\mathrm{LGL}}$ and $\Lambda_{n}^{\mathrm{CGL}}$ to find that the former is superior to the more commonly used Chebyshev-Gauss-Lobatto points for any practical value of $n$, while it is superior to the Legendre-Gauss-Lobatto points only for small $n$. An attractive property of $\Pi_{n}^{\mathrm{UGL}}$ is, however, that it is given in explicit form contrary to $\Pi_{n}^{\mathrm{LGL}}$.

In the present section we have summarized and extended the results for interpolation on nodal sets with particular emphasis on nodal sets appearing as solutions to an electrostatic problem and related to the symmetric Jacobi polynomials. The results may most easily be summarized as

$$
\Lambda_{n}^{\mathrm{Eq}}>\Lambda_{n}^{\mathrm{CG}}>\Lambda_{n}^{\mathrm{CGL}}>\Lambda_{n}^{\mathrm{LGL}} \sim \Lambda_{n}^{\mathrm{UGL}}>\Lambda_{n}^{\mathrm{ECG}},
$$

which we believe is valid for all practical values of $n$ in the context of spectral methods and is consistent with results put forward in [13, 28].

Although there remain many open questions when considering interpolation in $\mathrm{S}^{1}$ using Jacobi-Gauss-Lobatto nodal sets, it seems clear, however, that some of the nodal sets obtainable through the solution of an electrostatic problem are very close to what is theoretically possible.

4. Interpolation points in $\mathbf{S}^{2}$ using electrostatics. The problem of polynomial interpolation in $S^{2}$ is considerably more complex than in $S^{1}$ and very little is known. Hence, in the present work we shall focus our attention on polynomial interpolation of functions defined on an equilateral triangle, $\mathrm{S}^{2}$, as pictured in Fig. 2.

In the present context, it is convenient to use barycentric coordinates. Let us denote $\boldsymbol{v}_{i}, i \in[1,3]$, as the three vertices; i.e., in this case $\boldsymbol{v}_{1}=(-1,0), \boldsymbol{v}_{2}=(0, \sqrt{3})$, and $\boldsymbol{v}_{3}=(1,0)$, such that any $\boldsymbol{x} \in \mathrm{S}^{2}$ can be expressed using a convex combination of the barycentric coordinates, $\left(b_{1}, b_{2}, b_{3}\right)$, as

$$
\boldsymbol{x}=\sum_{i=1}^{3} \boldsymbol{v}_{i} b_{i} \text { and } \sum_{i=1}^{3} b_{i}=1
$$

and $0 \leq b_{i} \leq 1$. 
We introduce the polynomial space, $\mathrm{P}_{n}^{2}=\operatorname{span}\left\{p_{i j}(\boldsymbol{x})=x^{i} y^{j} ; i, j \geq 0 ; i+j \leq n\right\}$. The basis functions spanning the polynomial space may conveniently be thought of as

$\begin{array}{ccccccc} & & & 1 & & & \\ & & x & & y & & \\ x^{3} & x^{2} & & x y & & y^{2} & \\ & & x^{2} y & & y^{2} x & & y^{3} \\ & & & \vdots & & & \end{array}$

Estimation of the Lebesgue constant for interpolation in $\mathrm{S}^{2}$ is extremely difficult, and, only for the equidistant grid in barycentric coordinates given as

$$
\Pi_{n}^{\mathrm{Eq}}=\left\{\boldsymbol{x}_{i} \mid\left(b_{1}, b_{2}, b_{3}\right)=\left(1-\frac{k+l}{n}, \frac{k}{n}, \frac{l}{n}\right) ; k \geq 0, l \geq 0, k+l \leq n\right\}_{i=1}^{N},
$$

do results exist. We recall that $n$ represents the order of the polynomial approximation and also signifies the order of the polynomial employed along the edges. The explicit expression for the interpolating Lagrange polynomial, $L_{i}\left(\Pi_{n}^{\mathrm{Eq}}, \boldsymbol{x}\right)$, is given in [29], where the bound for the Lebesgue constant, $\Lambda_{n}^{\mathrm{Eq}}$, is derived also.

THEOREM 4.1. The Lebesgue constant, $\Lambda_{n}^{\mathrm{Eq}}$, using the nodal set, $\Pi_{n}^{\mathrm{Eq}}$, for polynomial interpolation in $\mathrm{S}^{2}$ is bounded as

$$
\Lambda_{n}^{\mathrm{Eq}} \leq\left(\begin{array}{c}
2 n-1 \\
n
\end{array}\right)
$$

As for interpolation along the line, we observe extremely rapid growth of $\Lambda_{n}^{\mathrm{Eq}}$, essentially rendering this nodal set useless for high-order polynomial interpolation. For reasons of comparison, we list the Lebesgue constants for $n \in[1,16]$ in Table 2 . The question naturally raises as to whether it is possible to obtain nodal sets better suited than $\Pi_{n}^{\mathrm{Eq}}$ for interpolation in $\mathrm{S}^{2}$.

This question was first addressed in [29] by realizing from (2) that a good nodal set could be obtained by maximizing the Vandermonde determinant, |VDM|, much like the procedure that leads to the Legendre-Gauss-Lobatto points discussed previously. However, the study was quite restricted and nodal sets were computed only for $n \leq 7$.

Recently, a more thorough study was published in [27], where nodal sets, resulting in a significantly slower growth of the Lebesgue constant than obtained for $\Pi_{n}^{\mathrm{Eq}}$, were obtained by minimizing over the $L_{2}$-norm of the Lebesgue function for $n \in[2,13]$. As is evident from the computed Lebesgue constants, $\Lambda_{n}^{\mathrm{Opt}}$, as reproduced in Table 2, the suggested procedure yields very good nodal sets, although there is no way one can know whether these are the optimal sets. Indeed, as we will show shortly, at least for $n \leq 5$ is it possible to find sets with a smaller Lebesgue constant. However, the main reason for our interest in obtaining alternative nodal sets is the fact that those given in [27] do not have nodal distributions along the edges that can be identified as JacobiGauss-Lobatto quadrature points. As we have argued previously, this latter property is advantageous if one wishes to apply the polynomials on the simplex in connection with spectral methods which are almost exclusively based on Jacobi polynomials.

Inspired by the success of polynomial interpolation in $\mathrm{S}^{1}$, when using nodal sets appearing as solutions to an electrostatic problem, we now propose to consider the following scenario.

Assume that the edges of the simplex, $\mathrm{S}^{2}$, labeled $\mathrm{I}_{l}$ as illustrated in Fig. 2, are fragments of a continuous line charge with the charge density, $\rho_{l}^{L}>0$. Consider now 
an arbitrary edge, $\left.\right|_{l}$, connecting the two vertices, $\boldsymbol{v}_{a}=\left(x_{a}, y_{a}\right)$ and $\boldsymbol{v}_{b}=\left(x_{b}, y_{b}\right)$. The electrostatic force, $\boldsymbol{F}_{l}(\boldsymbol{x})$, from the line acting on a particle with the charge, $\rho_{p}$, held at the position $\boldsymbol{x}$, is then given as $\boldsymbol{F}_{l}^{L}(\boldsymbol{x})=\rho_{p} \boldsymbol{E}_{l}^{L}(\boldsymbol{x})=-\rho_{p} \nabla \phi_{l}^{L}$, where $\phi_{l}^{L}$ is the electrostatic potential from the edge, $\mathrm{I}_{l}$. Contrary to the approach used for $S^{1}$, where the potential is logarithmic, we assume the potential to be algebraic, such that the potential from the line fragment at $\boldsymbol{x}$ is given as

$$
\phi_{l}^{L}(\boldsymbol{x})=\rho_{l}^{L} \int_{0}^{1} \frac{1}{\left|\boldsymbol{x}-\boldsymbol{x}_{L}\right|} d t,
$$

where $\boldsymbol{x}_{L}=\boldsymbol{v}_{a}+t\left(\boldsymbol{v}_{b}-\boldsymbol{v}_{a}\right), t \in[0,1]$, represents the edge. It is straightforward to perform this integration analytically.

We also assume that a number, $N_{p}$, of unit mass charges, $\rho_{p}$, are allowed to move freely inside the simplex, mutually interacting according to the potential

$$
\phi\left(\boldsymbol{x}_{i}, \boldsymbol{x}_{j}\right)=\frac{\rho_{p}^{2}}{\left|\boldsymbol{x}_{i}-\boldsymbol{x}_{j}\right|} .
$$

Let us now propose to consider the following problem.

Problem: Let the line charge density be given as $\rho_{l}^{L}>0$. Assume also that $N_{p}$ unit mass charges with unit charge, $\rho_{p}=1$, are allowed to move freely inside the simplex. What is the steady state position of the charges that minimizes the electrostatic energy

$$
W\left(\boldsymbol{x}_{1}, \ldots, \boldsymbol{x}_{N_{p}}\right)=\sum_{i=1}^{N_{p}}\left(\sum_{l=1}^{3} \phi_{l}^{L}\left(\boldsymbol{x}_{i}\right)+\frac{1}{2} \sum_{\substack{j=1 \\ j \neq i}}^{N_{p}} \phi\left(\boldsymbol{x}_{i}, \boldsymbol{x}_{j}\right)\right) .
$$

The problem is clearly very similar to the one originally put forward by Stieltjes [1, 2], however, with significantly more complexity.

Contrary to the $m=1$ case, we have no prior knowledge of a proper choice of the line charge density of the line, $\rho_{l}^{L}$, in order to obtain nodal sets well suited for polynomial interpolation. Indeed, we will later use this free parameter for optimizing the nodal sets. However, based on the knowledge gained from $S^{1}$, we believe that the most appropriate choice is $\rho_{1}^{L}=\rho_{2}^{L}=\rho_{3}^{L}=\rho^{L}$; i.e., the external potential field imposed by the line charges is symmetric.

As the aim is to construct nodal sets well suited for approximating partial differential equations in a multidomain setting, we require that the edges contain interpolation points. However, the specification of these points is at this point undetermined.

We also recall that the aim is to construct interpolating polynomials with the edge polynomials having the same order as that of the global interpolating polynomials; i.e., the total number of nodal points should be related to the number of freely moving charges as $N_{p}=N_{n}^{2}-3 n$, where $3 n$ is the number of nodal points reserved for the edges. This value of $N_{p}$ allows for the construction of a complete basis as discussed in section 2 .

We have chosen to approach the problem by solving it numerically as an $N$-body problem by integrating Newtons second law as

$$
\ddot{\boldsymbol{x}}_{i}=-\left(\sum_{l=1}^{3} \nabla \phi_{l}^{L}\left(\boldsymbol{x}_{i}\right)+\sum_{\substack{j=1 \\ j \neq i}}^{N_{p}} \nabla \phi\left(\boldsymbol{x}_{i}, \boldsymbol{x}_{j}\right)\right)-\varepsilon \dot{\boldsymbol{x}}_{i},
$$


where the last term, $\dot{\boldsymbol{x}}_{i}$, corresponds to a small friction in order to make the problem slightly dissipative. This equation is advanced in time using a 7(6) embedded Nyström-Runge-Kutta scheme with error control [30]. The resulting solution is passed on as an initial guess to a nonlinear solver to find the true steady state solution. We have used an algorithm based on a modification of Powell's hybrid algorithm [31].

The single most important remaining point is how to choose the initial conditions. Finding the global minimum of the energy function is in general extremely complicated, in particular for increasing $N_{p}$. However, since we are interested in solutions suitable for polynomial interpolation, we restrict our attention to steady state solutions that possess a high degree of symmetry. Inside the simplex, we consider solutions that are constructed of three symmetry patterns [27]. A charge can be situated at the center of the triangle, termed a 1-symmetry; it can be positioned along one of the three meridians, termed a 3 -symmetry; or it can be inside one of the six subtriangles bounded by the symmetry axes, referred to as a 6 -symmetry. If we denote the number of charges with a 1-symmetry, $n_{1}$, the number of charges with a 3 -symmetry, $n_{3}$, and those with a 6 -symmetry as $n_{6}$, the total number of charges is then obtained as $N_{p}=(n-1)(n-2) / 2=n_{1}+3 n_{3}+6 n_{6}$.

The integer solution to this equation is nonunique for $n>4$. Different solutions correspond to different symmetry patterns inherent in the distribution of the charges. We have used these initial symmetry patterns to construct the initial conditions and then searched for the specific symmetry pattern that results in a steady state solution with the minimum energy among all the possible symmetry patterns for a given $n$.

Having established the computational framework, let us now return to the two outstanding problems: specification of the line charge, $\rho^{L}$, and the distribution of the nodes along the edges. As mentioned previously, we are primarily interested in having a distribution of nodal points along the edges that is related to Jacobi-Gauss-Lobatto quadrature points; i.e., they are uniquely determined by the parameter, $\alpha$, of the Jacobi polynomial. At this point we realize that the problem of finding the best nodal distribution inside the simplex is reduced to a two-parameter minimization problem in $\rho^{L}$ and $\alpha$. This is much like the original problem, however, with the unfortunate difference that in $S^{2}$ we do not know how they are related or even if they are.

In the following we have used the approach outlined above to find symmetric, steady state solutions to the electrostatic problem. However, doing a full twodimensional optimization is out of reach and we have restricted our attention to a few special cases of $\alpha$ that are most interesting within the present context.

For a fixed value of $\alpha$, the problem is reduced to a one-dimensional minimization problem in $\rho^{L}$, which is feasible. In all problems we find that $\rho^{L} \sim \mathcal{O}(1)$ but varies slightly with $n$ as well as with $\alpha$.

As the most natural choice of $\alpha$ in relation to spectral methods, we have computed the nodal sets for $\alpha=-1 / 2$, corresponding to the Chebyshev-Gauss-Lobatto nodal set along the edge. In Table 2, we give the corresponding Lebesgue constant, $\Lambda_{n}^{\text {CGL }}$, for increasing $n$, confirming the soundness of the electrostatic approach. Indeed, we observe that the computed nodal sets are very good as compared to the corresponding equidistant nodal set, and they also compare well with the nodal sets presented in [27], indeed being superior for small values of $n$. In Appendix A we give the barycentric coordinates for the computed nodal sets for $n \in[2,16]$.

Another widely used nodal set for spectral methods is based on the LegendreGauss-Lobatto nodal set, i.e., corresponding to $\alpha=0$. We have obtained nodal sets with the Legendre-Gauss-Lobatto nodal set along the edges and the corresponding 
TABLE 2

Lebesgue constants for various choices of nodal sets in the simplex. Listed are the computed Lebesgue constants for the nodal set, $\Lambda_{n}^{\mathrm{JGL}, \alpha}$, with the optimal Jacobi-Gauss-Lobatto quadrature points along the edges. $\Lambda_{n}^{\mathrm{LGL}}$ and $\Lambda_{n}^{\mathrm{CGL}}$ represent the Lebesgue constants for the nodal sets with the Legendre-Gauss-Lobatto and Chebyshev-Gauss-Lobatto nodes along the edges, respectively. For comparison, we also give the Lebesgue constants, $\Lambda_{n}^{\mathrm{Opt}}$, for the nodal sets presented in [27] and the Lebesgue constants, $\Lambda_{n}^{\mathrm{Eq}}$, for the equidistant nodal set, $\Pi_{n}^{\mathrm{Eq}}$.

\begin{tabular}{||r|r||c|c|c||c|c||}
\hline$n$ & $N_{n}^{2}$ & $\Lambda_{n}^{\mathrm{JGL}, \alpha}$ & $\Lambda_{n}^{\mathrm{LGL}}$ & $\Lambda_{n}^{\mathrm{CGL}}$ & $\Lambda_{n}^{\mathrm{Opt}}$ & $\Lambda_{n}^{\mathrm{Eq}}$ \\
\hline 1 & 3 & 1.0000 & 1.0000 & 1.0000 & 1.0000 & 1.0000 \\
2 & 6 & 1.6667 & 1.6667 & 1.6667 & 1.6667 & 1.6667 \\
3 & 10 & 2.1299 & 2.1125 & 2.1150 & 2.1115 & 2.2698 \\
4 & 15 & 2.6033 & 2.5878 & 2.6050 & 2.6920 & 3.4748 \\
\hline 5 & 21 & 3.2171 & 3.1958 & 3.2096 & 3.3010 & 5.4522 \\
6 & 28 & 4.1291 & 4.0752 & 4.0736 & 3.7910 & 8.7477 \\
7 & 36 & 4.8848 & 4.7753 & 4.7846 & 4.3908 & 14.345 \\
8 & 45 & 6.0127 & 5.8518 & 5.8786 & 5.0893 & 24.007 \\
\hline 9 & 55 & 7.1075 & 6.8717 & 6.9174 & 5.9181 & 40.923 \\
10 & 66 & 8.371 & 8.4412 & 8.3963 & 7.0851 & 70.891 \\
11 & 78 & 10.603 & 10.075 & 10.091 & 8.3383 & 124.53 \\
12 & 91 & 13.550 & 12.628 & 12.517 & 10.082 & 221.41 \\
\hline 13 & 105 & - & - & 15.336 & 12.046 & 397.70 \\
14 & 120 & - & - & 22.184 & - & 720.70 \\
15 & 136 & - & - & 29.691 & - & 1315.9 \\
16 & 153 & - & - & 41.726 & - & 2418.5 \\
\hline
\end{tabular}

Lebesgue constants, $\Lambda_{n}^{\mathrm{LGL}}$, are also given in Table 2. Not surprisingly, we observe that these nodal sets seem to be slightly better than those related to the Chebyshev nodal sets, which is consistent with the findings in $\mathrm{S}^{1}$. The barycentric coordinates of the computed nodal sets are given in Appendix B, although we give them only for $n \in[2,12]$. For larger values of $n$ the optimal interior nodal sets are virtually identical to those obtained when using the Chebyshev-Gauss-Lobatto quadrature points along the edges since the contribution of the edges to the value of the Lebesgue constant becomes less and less significant for increasing $n$. Consequently, the nodal sets obtained when optimizing in $\rho^{L}$ for different values of $\alpha$ are very similar for large $n$. We therefore advocate using the nodal sets given in Appendix A for $\alpha=0$ and $n>12$ as well.

For comparison, we also give the Lebesgue constant, $\Lambda_{n}^{\mathrm{JGL}, \alpha}$, for nodal sets optimized for use with the optimal one-dimensional Jacobi-Gauss-Lobatto nodal set along the edges and in Table 2, showing that both of the former nodal sets are superior to this latter set, which we therefore discard.

Regarding the value of the optimal line charge, we find that for small values of $n$, $\rho^{L}$ is highly dependent on the choice of $\alpha$. However, for increasing $n$, i.e., $n>10$, the optimal value of $\rho^{L}$ is virtually independent on $\alpha$ and attains a value of $\rho^{L} \simeq 0.75$.

In Fig. 3 we illustrate the resulting nodal sets for $\alpha=-0.5$ and various values of $n$, clearly showing the high degree of symmetry characterizing the nodal sets.

5. Concluding remarks. In this paper we have exploited the remarkable connection between solutions to problems of electrostatics and nodal sets well suited for polynomial interpolation along the line. The emphasis has been on nodal sets constructed from the Jacobi-Gauss-Lobatto quadrature points and we have shown how they relate to the solution of a problem of electrostatics. Based on extensive numerical evidence we have also conjectured several estimates on the variation of the Lebesgue constant with $n$ for the Jacobi-Gauss-Lobatto quadrature points, for which only few 


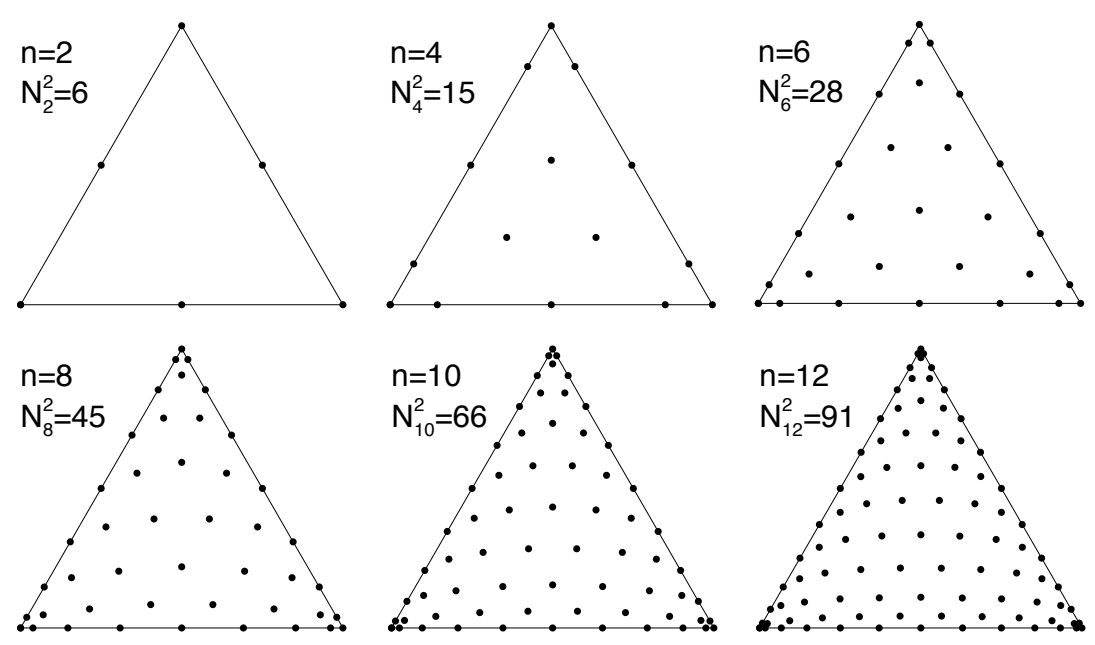

FIG. 3. Nodal sets in the equilateral triangle with Chebyshev-Gauss-Lobatto nodal sets along the edges.

analytical results exist. In particular, our results indicate that the nodal sets based on the Gauss-Lobatto quadrature points of the Chebyshev polynomial of the second kind is the best choice among the Jacobi polynomials for which there exists a closed form expression of the nodal set. This suggests that the Chebyshev polynomial of the second kind, rather than the extensively used Chebyshev polynomials of the first kind, could be used with advantage as the basis functions for solving partial differential equations using spectral methods, at least for small values of $N$ where fast transforms are of less importance.

The possibility of formulating an electrostatic problem leading to very good nodal sets for polynomial interpolation along the line inspired us to attempt a similar approach in a 2-simplex with the emphasis being on an equilateral triangle. We have formulated a natural extension of the original approach and found that the solution of that electrostatic problem leads to nodal sets which are very close to the best known sets, thus confirming that the electrostatic analogy seems to carry over to the multidimensional case, provided the problem is properly posed. Indeed, there are many ways this can be done, in particular with respect to the treatment of the bounding edges, where we chose the simplest possible approach in order to minimize the computational workload required to find the sought-after steady state solutions. However, our results clearly show that there is a connection between the solution to problems of electrostatics and nodal sets well suited for polynomial interpolation in the multidimensional simplex. It is our belief that a very similar approach can be applied for finding suitable nodal sets in a higher-dimensional simplex as well.

When computing the nodal sets for polynomial interpolation in the equilateral triangle, we chose to restrict the attention to solutions where the nodes along the edges were distributed as Gauss-Lobatto quadrature points of Chebyshev or Legendre polynomials. The reason for this is that Chebyshev and Legendre polynomials are the most commonly used polynomial basis for obtaining solutions to partial differential equations through spectral methods. Consequently, the computed nodal sets can easily be combined with more conventional multidomain spectral methods or 
TABLE 3

\begin{tabular}{|c|c|c|c|c|c|c|}
\hline$n$ & $n_{1}$ & $n_{2}$ & $n_{3}$ & $b_{1}$ & $b_{2}$ & $b_{3}$ \\
\hline 3 & 1 & & & 0.3333333333 & 0.3333333333 & 0.3333333333 \\
\hline 4 & & 1 & & 0.2410021998 & 0.2410021998 & 0.5179956004 \\
\hline \multirow[t]{2}{*}{5} & \multirow{2}{*}{\multicolumn{2}{|c|}{2}} & & 0.1591570023 & 0.1591570023 & 0.6816859954 \\
\hline & & & & 0.4099016620 & 0.4099016620 & 0.1801966760 \\
\hline \multirow[t]{3}{*}{6} & \multirow[t]{3}{*}{1} & \multirow{3}{*}{1} & & 0.3333333333 & 0.3333333333 & 0.3333333333 \\
\hline & & & & 0.1048904342 & 0.1048904342 & 0.7902191316 \\
\hline & & & 1 & 0.3095036860 & 0.5582114022 & 0.1322849118 \\
\hline \multirow[t]{4}{*}{7} & & \multirow[t]{4}{*}{3} & & 0.0666479037 & 0.0666479037 & 0.8667041924 \\
\hline & & & & 0.4474963910 & 0.4474963910 & 0.1050072180 \\
\hline & & & & 0.2606379453 & 0.2606379453 & 0.4787241094 \\
\hline & & & 1 & 0.2328951264 & 0.6750593997 & 0.0920454739 \\
\hline \multirow[t]{5}{*}{8} & & \multirow[t]{5}{*}{3} & & 0.0467325482 & 0.0467325482 & 0.9065349036 \\
\hline & & & & 0.2031909379 & 0.2031909379 & 0.5936181242 \\
\hline & & & & 0.3906323571 & 0.3906323571 & 0.2187352858 \\
\hline & & & 2 & 0.3618069634 & 0.5544121321 & 0.0837809045 \\
\hline & & & & 0.1799524415 & 0.7523326932 & 0.0677148653 \\
\hline \multirow[t]{7}{*}{9} & 1 & \multirow{7}{*}{3} & & 0.3333333333 & 0.3333333333 & 0.3333333333 \\
\hline & & & & 0.0354284515 & 0.0354284515 & 0.9291430970 \\
\hline & & & & 0.4641239296 & 0.4641239296 & 0.0717521408 \\
\hline & & & & 0.1632684184 & 0.1632684184 & 0.6734631632 \\
\hline & & & 3 & 0.2965866112 & 0.6351919517 & 0.0682214371 \\
\hline & & & & 0.1437685751 & 0.8034472485 & 0.0527841764 \\
\hline & & & & 0.3225938344 & 0.4969468296 & 0.1804593360 \\
\hline \multirow[t]{8}{*}{10} & & \multirow[t]{8}{*}{4} & & 0.0267617465 & 0.0267617465 & 0.9464765070 \\
\hline & & & & 0.1333154550 & 0.1333154550 & 0.7333690900 \\
\hline & & & & 0.4230209738 & 0.4230209738 & 0.1539580524 \\
\hline & & & & 0.2834641787 & 0.2834641787 & 0.4330716426 \\
\hline & & & 4 & 0.3934497870 & 0.5469389728 & 0.0596112402 \\
\hline & & & & 0.2464304982 & 0.6986850110 & 0.0548844908 \\
\hline & & & & 0.1165696792 & 0.8422416956 & 0.0411886252 \\
\hline & & & & 0.2707661899 & 0.5807728926 & 0.1484609175 \\
\hline \multirow[t]{10}{*}{11} & & \multirow[t]{10}{*}{5} & & 0.0205770993 & 0.0205770993 & 0.9588458014 \\
\hline & & & & 0.4745463805 & 0.4745463805 & 0.0509072390 \\
\hline & & & & 0.1106491928 & 0.1106491928 & 0.7787016144 \\
\hline & & & & 0.2422912395 & 0.2422912395 & 0.5154175210 \\
\hline & & & & 0.3764104927 & 0.3764104927 & 0.2471790146 \\
\hline & & & 5 & 0.3362467835 & 0.6143637919 & 0.0493894246 \\
\hline & & & & 0.2075057639 & 0.7480004251 & 0.0444938110 \\
\hline & & & & 0.0961598440 & 0.8711975595 & 0.0326425965 \\
\hline & & & & 0.3648517435 & 0.5058451075 & 0.1293031490 \\
\hline & & & & 0.2299937315 & 0.6463450688 & 0.1236611997 \\
\hline \multirow[t]{12}{*}{12} & 1 & \multirow{12}{*}{4} & & 0.3333333333 & 0.3333333333 & 0.3333333333 \\
\hline & & & & 0.0157664060 & 0.0157664060 & 0.9684671880 \\
\hline & & & & 0.0927641836 & 0.0927641836 & 0.8144716328 \\
\hline & & & & 0.4451055330 & 0.4451055330 & 0.1097889340 \\
\hline & & & & 0.2092594550 & 0.2092594550 & 0.5814810900 \\
\hline & & & 7 & 0.4144591572 & 0.5428398510 & 0.0427009918 \\
\hline & & & & 0.2891782274 & 0.6704310002 & 0.0403907724 \\
\hline & & & & 0.1766396330 & 0.7874226099 & 0.0359377571 \\
\hline & & & & 0.0801575546 & 0.8939792746 & 0.0258631708 \\
\hline & & & & 0.3176588844 & 0.5750391576 & 0.1073019580 \\
\hline & & & & 0.1970549373 & 0.6986253330 & 0.1043197297 \\
\hline & & & & 0.3292289528 & 0.4566354051 & 0.2141356421 \\
\hline
\end{tabular}

even provide the basic building block for a triangular spectral element method with unstructured grids interior to the triangle [32].

Appendix A: Nodal matrix for the triangle with Chebyshev-GaussLobatto nodal sets along the edges. In Tables $3-5$ we give the barycentric co- 
TABLE 4

\begin{tabular}{|c|c|c|c|c|c|c|}
\hline$n$ & $n_{1}$ & $n_{2}$ & $n_{3}$ & $b_{1}$ & $b_{2}$ & $b_{3}$ \\
\hline \multirow[t]{14}{*}{13} & \multirow{14}{*}{\multicolumn{2}{|c|}{6}} & \multirow{14}{*}{8} & 0.0125158559 & 0.0125158559 & 0.9749682882 \\
\hline & & & & 0.4814041934 & 0.4814041934 & 0.0371916132 \\
\hline & & & & 0.0791069148 & 0.0791069148 & 0.8417861704 \\
\hline & & & & 0.1831015613 & 0.1831015613 & 0.6337968774 \\
\hline & & & & 0.4068245409 & 0.4068245409 & 0.1863509182 \\
\hline & & & & 0.2954002632 & 0.2954002632 & 0.4091994736 \\
\hline & & & & 0.3615540438 & 0.6022119116 & 0.0362340446 \\
\hline & & & & 0.2513189999 & 0.7149811088 & 0.0336998913 \\
\hline & & & & 0.1523482839 & 0.8179762698 & 0.0296754463 \\
\hline & & & & 0.0680753374 & 0.9108476566 & 0.0210770060 \\
\hline & & & & 0.3944786597 & 0.5151527485 & 0.0903685918 \\
\hline & & & & 0.2767319260 & 0.6304802457 & 0.0927878283 \\
\hline & & & & 0.1706952037 & 0.7399484931 & 0.0893563032 \\
\hline & & & & 0.2915934740 & 0.5215057084 & 0.1869008176 \\
\hline \multirow[t]{16}{*}{14} & & \multirow[t]{16}{*}{6} & \multirow{16}{*}{10} & 0.4587977340 & 0.4587977340 & 0.0824045320 \\
\hline & & & & 0.3671908093 & 0.3671908093 & 0.2656183814 \\
\hline & & & & 0.2595748224 & 0.2595748224 & 0.4808503552 \\
\hline & & & & 0.1603773315 & 0.1603773315 & 0.6792453370 \\
\hline & & & & 0.0685768901 & 0.0685768901 & 0.8628462198 \\
\hline & & & & 0.0103478215 & 0.0103478215 & 0.9793043570 \\
\hline & & & & 0.9234057833 & 0.0588664441 & 0.0177277726 \\
\hline & & & & 0.8416660290 & 0.1330619104 & 0.0252720607 \\
\hline & & & & 0.7521462903 & 0.2191316332 & 0.0287220764 \\
\hline & & & & 0.6425344401 & 0.3256595455 & 0.0318060144 \\
\hline & & & & 0.5379997781 & 0.4292596705 & 0.0327405513 \\
\hline & & & & 0.4692799339 & 0.3610693351 & 0.1696507310 \\
\hline & & & & 0.7721478696 & 0.1494014554 & 0.0784506750 \\
\hline & & & & 0.5652822600 & 0.3505856755 & 0.0841320645 \\
\hline & & & & 0.6781794781 & 0.2494595411 & 0.0723609808 \\
\hline & & & & 0.5834887797 & 0.2550816171 & 0.1614296032 \\
\hline \multirow[t]{19}{*}{15} & 1 & \multirow{19}{*}{6} & \multirow{19}{*}{12} & 0.3333333333 & 0.3333333333 & 0.3333333333 \\
\hline & & & & 0.4852528832 & 0.4852528832 & 0.0294942336 \\
\hline & & & & 0.4255990820 & 0.4255990820 & 0.1488018360 \\
\hline & & & & 0.2320836842 & 0.2320836842 & 0.5358326316 \\
\hline & & & & 0.1405179457 & 0.1405179457 & 0.7189641086 \\
\hline & & & & 0.0599963506 & 0.0599963506 & 0.8800072988 \\
\hline & & & & 0.0085857218 & 0.0085857218 & 0.9828285564 \\
\hline & & & & 0.9337196169 & 0.0512926813 & 0.0149877018 \\
\hline & & & & 0.8617284617 & 0.1169291070 & 0.0213424313 \\
\hline & & & & 0.7794706504 & 0.1957424109 & 0.0247869387 \\
\hline & & & & 0.6834546988 & 0.2890601702 & 0.0274851310 \\
\hline & & & & 0.5886608293 & 0.3824865582 & 0.0288526124 \\
\hline & & & & 0.8006726315 & 0.1325472687 & 0.0667800998 \\
\hline & & & & 0.7134213061 & 0.2207559648 & 0.0658227291 \\
\hline & & & & 0.6135939037 & 0.3123505510 & 0.0740555453 \\
\hline & & & & 0.5171616793 & 0.4145995177 & 0.0682388030 \\
\hline & & & & 0.6286428956 & 0.2270147509 & 0.1443423535 \\
\hline & & & & 0.5244220875 & 0.3265860871 & 0.1489918254 \\
\hline & & & & 0.4324830342 & 0.3299898010 & 0.2375271649 \\
\hline
\end{tabular}

ordinates for the nodal sets optimized for use with Chebyshev-Gauss-Lobatto nodal sets of order $n$ along the edges; i.e., they are distributed as the zeros of the polynomial, $\left(1-x^{2}\right) T_{n}^{\prime}(x)$. Only the nodes interior to the simplex are given, and nodes processing symmetries are only given once. The remaining nodes can be found by permutations of the barycentric coordinates. 
TABLE 5

\begin{tabular}{|c|c|c|c|c|c|c|}
\hline$n$ & $n_{1}$ & $n_{2}$ & $n_{3}$ & $b_{1}$ & $b_{2}$ & $b_{3}$ \\
\hline \multirow[t]{21}{*}{16} & & 7 & \multirow{21}{*}{14} & 0.4692881367 & 0.4692881367 & 0.0614237267 \\
\hline & & & & 0.3932305456 & 0.3932305456 & 0.2135389089 \\
\hline & & & & 0.3025577746 & 0.3025577746 & 0.3948844509 \\
\hline & & & & 0.2078492229 & 0.2078492229 & 0.5843015542 \\
\hline & & & & 0.1284932701 & 0.1284932701 & 0.7430134598 \\
\hline & & & & 0.0529388222 & 0.0529388222 & 0.8941223556 \\
\hline & & & & 0.0072437614 & 0.0072437614 & 0.9855124772 \\
\hline & & & & 0.9419105656 & 0.0452492885 & 0.0128401458 \\
\hline & & & & 0.8772044870 & 0.1042200005 & 0.0185755125 \\
\hline & & & & 0.8060459745 & 0.1727546471 & 0.0211993784 \\
\hline & & & & 0.7198500717 & 0.2560058606 & 0.0241440677 \\
\hline & & & & 0.6256312013 & 0.3487602527 & 0.0256085459 \\
\hline & & & & 0.5343544917 & 0.4393591499 & 0.0262863584 \\
\hline & & & & 0.8214366546 & 0.1173927115 & 0.0611706338 \\
\hline & & & & 0.7464832409 & 0.1959218281 & 0.0575949309 \\
\hline & & & & 0.6578452111 & 0.2860606787 & 0.0560941102 \\
\hline & & & & 0.5620632423 & 0.3743524772 & 0.0635842805 \\
\hline & & & & 0.6657283975 & 0.1263493158 & 0.2079222867 \\
\hline & & & & 0.5755509622 & 0.2921362629 & 0.1323127749 \\
\hline & & & & 0.4806452274 & 0.3853613574 & 0.1339934152 \\
\hline & & & & 0.4883520007 & 0.2978352313 & 0.2138127680 \\
\hline
\end{tabular}

TABLE 6

\begin{tabular}{|c|ccc|ccc|}
\hline$n$ & $n_{1}$ & $n_{2}$ & $n_{3}$ & $b_{1}$ & $b_{2}$ & $b_{3}$ \\
\hline 3 & 1 & & & 0.3333333333 & 0.3333333333 & 0.3333333333 \\
\hline 4 & & 1 & & 0.2371200168 & 0.2371200168 & 0.5257599664 \\
\hline 5 & & 2 & & 0.1575181512 & 0.1575181512 & 0.6849636976 \\
& & & & 0.4105151510 & 0.4105151510 & 0.1789696980 \\
\hline 6 & 1 & & & 0.3333333333 & 0.3333333333 & 0.3333333333 \\
& & 1 & & 0.1061169285 & 0.1061169285 & 0.7877661430 \\
& & & 1 & 0.3097982151 & 0.5569099204 & 0.1332918645 \\
\hline 7 & & 3 & & 0.0660520784 & 0.0660520784 & 0.8678958432 \\
& & & & 0.4477725053 & 0.4477725053 & 0.1044549894 \\
& & & & 0.2604038024 & 0.2604038024 & 0.4791923952 \\
& & & 1 & 0.2325524777 & 0.6759625951 & 0.0914849272 \\
\hline 8 & & 3 & & 0.0469685351 & 0.0469685351 & 0.9060629298 \\
& & & & 0.2033467796 & 0.2033467796 & 0.5933064408 \\
& & & 2 & 0.3905496216 & 0.3905496216 & 0.2189007568 \\
& & & & 0.3617970895 & 0.5541643672 & 0.0840385433 \\
& & & & 0.33333333333 & 0.3333333333 & 0.3333333333 \\
& 1 & \multirow{2}{*}{3} & & 0.0355775717 & 0.0355775717 & 0.9288448566 \\
& & & & 0.4640303025 & 0.4640303025 & 0.0719393950 \\
& & & & 0.1633923069 & 0.1633923069 & 0.6732153862 \\
& & & 3 & 0.2966333890 & 0.6349633653 & 0.0684032457 \\
& & & & 0.1439089974 & 0.8031490682 & 0.0529419344 \\
& & & & 0.3225890045 & 0.4968009397 & 0.1806100558 \\
\hline
\end{tabular}

Appendix B: Nodal matrix for the triangle with Legendre-GaussLobatto nodal sets along the edges. In Tables 6 and 7, we give the barycentric coordinates for the nodal sets optimized for use with Legendre-Gauss-Lobatto nodal sets of order $n$ along the edges; i.e., they are distributed as the zeros of the polynomial, $\left(1-x^{2}\right) P_{n}^{\prime}(x)$. Only the nodes interior to the simplex are given and nodes processing symmetries are only given once. The remaining nodes can be found by permutations of the barycentric coordinates. 
TABLE 7

\begin{tabular}{|c|c|c|c|c|c|c|}
\hline$n$ & $n_{1}$ & $n_{2}$ & $n_{3}$ & $b_{1}$ & $b_{2}$ & $b_{3}$ \\
\hline \multirow[t]{8}{*}{10} & \multirow{8}{*}{\multicolumn{2}{|c|}{4}} & \multirow{8}{*}{4} & 0.0265250690 & 0.0265250690 & 0.9469498620 \\
\hline & & & & 0.1330857076 & 0.1330857076 & 0.7338285848 \\
\hline & & & & 0.4232062312 & 0.4232062312 & 0.1535875376 \\
\hline & & & & 0.2833924371 & 0.2833924371 & 0.4332151258 \\
\hline & & & & 0.3934913008 & 0.5472380443 & 0.0592706549 \\
\hline & & & & 0.2462883939 & 0.6991456238 & 0.0545659823 \\
\hline & & & & 0.1163195333 & 0.8427538829 & 0.0409265838 \\
\hline & & & & 0.2707097521 & 0.5811217960 & 0.1481684519 \\
\hline \multirow[t]{10}{*}{11} & & \multirow[t]{10}{*}{5} & \multirow{10}{*}{5} & 0.0204278105 & 0.0204278105 & 0.9591443790 \\
\hline & & & & 0.4746683133 & 0.4746683133 & 0.0506633734 \\
\hline & & & & 0.1104863580 & 0.1104863580 & 0.7790272840 \\
\hline & & & & 0.2422268680 & 0.2422268680 & 0.5155462640 \\
\hline & & & & 0.3764778430 & 0.3764778430 & 0.2470443140 \\
\hline & & & & 0.3361965758 & 0.6146545276 & 0.0491488966 \\
\hline & & & & 0.2073828199 & 0.7483396985 & 0.0442774816 \\
\hline & & & & 0.0959885136 & 0.8715407373 & 0.0324707491 \\
\hline & & & & 0.3649298091 & 0.5060795425 & 0.1289906484 \\
\hline & & & & 0.2299163838 & 0.6466193522 & 0.1234642640 \\
\hline \multirow[t]{12}{*}{12} & 1 & \multirow{12}{*}{4} & \multirow{12}{*}{7} & 0.3333333333 & 0.3333333333 & 0.3333333333 \\
\hline & & & & 0.0156346170 & 0.0156346170 & 0.9687307660 \\
\hline & & & & 0.0926054449 & 0.0926054449 & 0.8147891102 \\
\hline & & & & 0.4452623516 & 0.4452623516 & 0.1094752968 \\
\hline & & & & 0.2091994115 & 0.2091994115 & 0.5816011770 \\
\hline & & & & 0.4145270405 & 0.5430117044 & 0.0424612551 \\
\hline & & & & 0.2890538328 & 0.6707932601 & 0.0401529071 \\
\hline & & & & 0.1765067641 & 0.7877603302 & 0.0357329057 \\
\hline & & & & 0.0799959921 & 0.8942989924 & 0.0257050155 \\
\hline & & & & 0.3177076680 & 0.5753716491 & 0.1069206829 \\
\hline & & & & 0.1969473706 & 0.6989024696 & 0.1041501598 \\
\hline & & & & 0.3292603428 & 0.4567841256 & 0.2139555316 \\
\hline
\end{tabular}

Acknowledgment. The author acknowledges many fruitful conversations with Prof. D. Gottlieb and Dr. C. B. Quillen, Brown University. Comments from Dr. M. Carpenter, NASA Langley Research Center, are likewise highly appreciated.

\section{REFERENCES}

[1] T. J. Stieltues, Sur quelques théorèmes d'algèbre, Comptes Rendus de l'Acadèmie des Sciences, 100 (1885), pp. 439-440.

[2] T. J. Stieltues, Sur les Polynômes de Jacobi, Comptes Rendus Acad. Sci., 100 (1885), pp. 620-622.

[3] G. Szegö, Orthogonal Polynomials, American Mathematical Society, 5th ed., Providence, RI, 1975 .

[4] C. Canuto, M. Y. Hussaini, A. Quarteroni, and T. A. Zang, Spectral Methods in Fluid Mechanics. Springer Series in Computational Physics, Springer-Verlag, Berlin, 1988.

[5] I. BABUŠKA AND M. SURI, The $p$ - and $h-p$ version of the finite element method. An overview, Comput. Methods Appl. Mech. Engrg., 80 (1990), pp. 5-26.

[6] A. PaterA, A spectral element method for fluid dynamics: Laminar flow in a channel, J. Comput. Phys., 54 (1984), pp. 468-488.

[7] R. P. Feinerman and D. J. Newman, Polynomial Approximation, Williams and Wilkins, Baltimore, MD, 1974.

[8] K. Chung AND T. YAO, On lattices admitting unique Lagrange interpolations, SIAM J. Numer. Anal., 14 (1977), pp. 735-743.

[9] N. J. Higham, Accuracy and Stability of Numerical Algorithms, SIAM, Philadelphia, PA, 1996.

[10] P. J. DAVIS, Interpolation and Approximation, Dover Publications, New York, 1975.

[11] T. J. Rivlin, An Introduction to the Approximation of Functions, Dover, New York, 1969.

[12] J. Szabados And P. VÉRTesi, Interpolation of Functions, World Scientific, River Edge, NJ, 1990. 
[13] F. W. Luttmann And T. J. Rivlin, Some numerical experiments in the theory of polynomial interpolation, IBM J. Res. Develop., 9 (1965), pp. 187-191.

[14] T. A. KILGore, A characterization of the Lagrange interpolation projection with minimal Tchebycheff norm, J. Approx. Theory, 24 (1978), pp. 273-288.

[15] C. De Boor And A. Pinkus, Proof of the conjecture of Bernstein and Erdós concerning the optimal nodes for polynomial interpolation, J. Approx. Theory, 24 (1978), pp. 289-303.

[16] J. R. Angelos, E. H. Kaufman JR., M. S. Henry, and T. D. Lenker, Optimal Nodes for Polynomial Interpolation, in Approximation Theory VI: Vol I, C. K. Chui, L. L. Schumaker, and J. D. Ward, eds., Academic Press, New York, 1989, pp. 17-20.

[17] P. ERdös, Problem and Results on the Theory of Interpolation. II, Acta Math. Acad. Sci. Hungar., 12 (1961), pp. 235-244.

[18] A. H. TURETskiI, The bounding of polynomials prescribed at equally distributed points, Proc. Pedag. Inst. Vitebsk., 3 (1940), pp. 117-127.

[19] A. SchöNHAGe, Fehlerfortpflanzung bei Interpolation, Numer. Math., 3 (1961), pp. 62-71.

[20] L. N. Trefethen and J. C. Weideman, Two results on polynomial interpolation in equally spaced points, J. Approx. Theory, 65 (1991), pp. 247-260.

[21] R. GÜnttner, Evaluation of Lebesgue constants, SIAM J. Numer. Anal., 17 (1980), pp. $512-520$

[22] P. N. Shivakumar And R. Wong, Asymptotic expansion of the Lebesgue constants associated with polynomial interpolation, Math. Comp., 39 (1982), pp. 195-200.

[23] A. ERdélyi, Higher transcendental functions, Vol. II, Robert Krieger, Melbourne, FL, 1953.

[24] J. H. McCabe And G. M. Phillips, On a Certain Class of Lebesgue Constants, BIT, 13 (1973), pp. 434-442.

[25] L. FEJÉR, Lagrangesche Interpolation und die Zugehörigen Konjugierten Punkte, Math. Ann., 106 (1932), pp. 1-55.

[26] B. Sundermann, Lebesgue Constants in Lagrangian Interpolation at the Fekete Points, Ergebnisbericht der Lehrstuhle Math III, VIII, 40, Univ. Dortmunn, Germany, 1980.

[27] Q. Chen And I. BABUŠKA, Approximate optimal points for polynomial interpolation of real functions in an interval and in a triangle, Comput. Methods Appl. Mech. Engrg., 128 (1995), pp. 405-417.

[28] L. BRutman, On the Lebesgue function for polynomial interpolation, SIAM J. Numer. Anal., 15 (1978), pp. 694-704.

[29] L. P. Bos, Bounding the Lebesgue function for interpolation in a simplex, J. Approx. Theory, 38 (1983), pp. 43-59.

[30] E. Hairer, S. P. Nørsett, And G. Wanner, Solving Ordinary Differential Equations I. Springer Series in Computational Mathematics 8, Springer-Verlag, Berlin, 1980.

[31] J. J. More, B. S. Garbow, and K. E. Hillstrom, User Guide for MINPACK-1, Report ANL-80-74, Argonne National Laboratory, Argonne, IL, 1980.

[32] J. S. Hesthaven and D. Gottlieb, Stable spectral methods for conservations laws on triangles with unstructured grids, SIAM J. Numer. Anal., 1997, submitted. 